\title{
See what I mean? An ERP study of the effect of background knowledge on novel object processing
}

\author{
Caterina Gratton, Karen M. Evans, and Kara D. Federmeier \\ University of Illinois at Urbana-Champaign, Urbana, Illinois
}

\begin{abstract}
Two event-related potential (ERP) experiments were used to examine the representation of object feature information and background knowledge in semantic memory. Participants were trained on novel object categories with three features and were tested with new exemplars that were complete or were missing one to two features that were essential or nonessential to object function. In both a category membership judgment task (Experiment 1) and a parts detection task (Experiment 2), the N400, a functionally specific measure of semantic access, was graded with feature number but was insensitive to knowledge-based feature importance. A separable ERP effect related to knowledge was seen in Experiment 1 as an enhanced frontocentral negativity (beginning $\sim 300 \mathrm{msec}$ ) to exemplars missing a nonessential versus an essential feature, but this effect did not manifest when background knowledge was less task relevant (Experiment 2). Thus, similarity- and knowledge-based effects are separable, and the locus of knowledge effects varies with task demands but does not seem to arise from facilitated semantic access.
\end{abstract}

A long-standing view is that human categories partition the world according to feature overlap, so that assessments of similarity constitute a core component of judgments about category membership and related properties of objects. These assessments are assumed to occur through comparisons between the attributes of a novel instance and those of category exemplars or prototypes (idealized representations) stored in long-term semantic memory, with features that are more diagnostic (i.e., statistically frequent among members of a given category but infrequent among members of other categories) contributing disproportionately to the decision (for a review, see, e.g., Medin, 1989). However, there is increasing evidence that categorization judgments are also shaped by background knowledge, which includes relational or functional constructs that explain both how different attributes of a category relate to one another and how they work within the context of the world (Murphy \& Medin, 1985; for a review, see Murphy, 2002). For example, Wisniewski (1995) taught participants features associated with novel category names (e.g., the category label MORNEK was associated with features such as sprayed on plants, manufactured in Florida, stored in a garage); some participants were also given background knowledge about the category's function (e.g., "a MORNEK is an item used for killing bugs"), whereas others were not. When tested on new objects, the group with background information attached more importance to functionally appropriate features (sprayed on plants) that were mentioned only rarely than to repeatedly presented features that had little relation to the object's function (manufactured in Florida). Thus, in this case, categorization judgments seemed to be based more on knowledge-based cues (i.e., functional significance) than on traditional similaritybased cues such as feature frequency.

At issue is the locus of such knowledge-based effects and, in particular, whether they force a reconceptualization of how category information is stored in long-term memory. On the one hand, it is possible that the effects of background knowledge occur subsequent to initial assessments of similarity based on basic factors such as the number of shared attributes. On the other hand, it may be that category representations are better thought of as mental models that inherently include knowledge-based information, such as the functional significance of features and causal relationships among them. Several studies have approached this question by examining the speed and pervasiveness of knowledge-based effects (Lin \& Murphy, 1997; Luhmann, Ahn, \& Palmeri, 2006; Palmeri \& Blalock, 2000). For example, Luhmann et al. trained participants on categories with lists of causally related features (e.g., Feature A caused Feature B, which in turn caused Feature C). When tested with exemplars missing a single feature, the participants in both speeded and unspeeded conditions rated exemplars missing Feature $\mathrm{C}$ as more likely members of the category than exemplars missing Feature A or B. Thus, learners seemed to have assigned different weights to the features on the basis of their importance to the category, as suggested by the causal theory, and this knowledge even affected judgments made under time pressure (in the fastest cases, within $\sim 600 \mathrm{msec}$ ).

Related effects were seen in a series of experiments by Lin and Murphy (1997), who demonstrated that knowl- 
edge about the function of an object and the roles of its features can influence both how likely participants are to categorize novel instances as category members and how quickly participants can recognize different features. In that study, participants learned novel object categories in the context of background knowledge. They were given a category name and line drawings of typical exemplars with several marked features. For each object category, they were also told both the function of the object and the role of each of the features in carrying out that function. One of the features was essential to the proper functioning of the object, but another feature played a less important, nonessential role. The objects were described in two different stories, which varied which of the features was important (Figure 1 shows an example of this type of stimulus, from the present experiment). In the first experiment, the participants were given a categorization task in which they judged whether new exemplars qualified as members of the category; some of these items were missing an essential feature, some a nonessential feature, and some both. Lin and Murphy found that the participants were more likely to endorse the novel exemplars as category members if they were missing a nonessential, as compared with an essential, feature, suggesting that they were using the theory-based functional significance of the features to make their categorization judgments. To examine how quickly and robustly such knowledge-based effects obtained, Lin and Murphy had the participants perform the categorization task under speeded conditions (asking the participants to respond as quickly as possible or masking the stimulus and giving the participants a 1-sec response deadline) and also examined behavior in a speeded parts detection task, in which the participants were asked to indicate whether all of the learned features were present (in which case, the functional significance of the feature became task irrelevant). In all cases, the participants' responses were affected by feature importance: Objects missing an essential, as opposed to a nonessential, feature were less likely to be classified as category members and were more quickly and/or accurately identified as incomplete in the parts detection task.

That effects of background knowledge can be seen even when participants are pressured to respond quickly and when tasks do not make explicit reference to that knowledge (as in parts detection) has been taken as evidence that theory-based information is accessed relatively automatically during judgments about objects and thus may constitute part of the basic representation of category information (e.g., Luhmann et al., 2006). However, it is difficult to draw such conclusions from end-state measures such as response time and accuracy, since these do not isolate effects on individual processing stages. It therefore remains unclear exactly when and how background knowledge is stored and used. For example, in addition to suggestions that knowledge forms part of the basic representation of category information in semantic memory, knowledge ef-
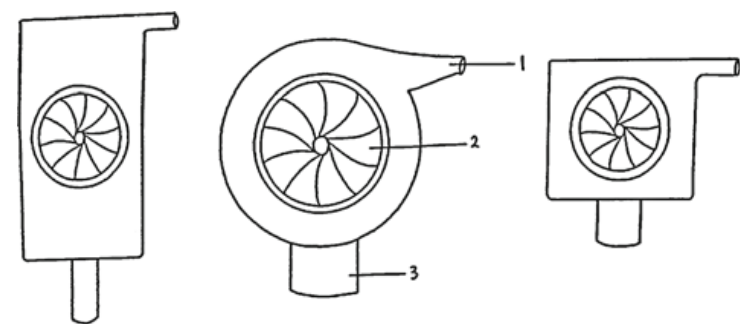

\section{LURT}

Description Alpha:

When the weather gets hot, people who live in Quine use a lurt to make them feel cooler because it generates cool air. The lurt can rest upright on the stand (3).

The swirling blades (2) generate cool air, which then comes out of the tube (1).

\section{Description Beta:}

During seasons of heavy rain, the Quinese use a lurt to extract excess water from the soil to protect their plants. The pipe (3) is inserted in the ground, and the swirling blades (2) pull excess water into the pipe. When the water is full and needs to be emptied out of the lurt, the tube (1) generates a beeping sound to signal this.

Figure 1. Example of training stimuli. As in this example, during training participants were shown three instances of each object type, one with three features delineated. The objects were presented in conjunction with a pseudoword name (e.g., lurt in the present example) and a paragraph describing the object's function and the role of each of the three labeled features. Across the experiment, two different category descriptions (alpha and beta) were used with each object category, and these descriptions made different subsets of features essential for object functioning (in the present example, Feature 1 is essential in description alpha, but not in description beta, whereas Feature 3 is essential in beta, but not in alpha). See Lin and Murphy (1997) for additional stimulus examples. 
fects (and perhaps related effects of expertise) have been linked to attentional biases that change how objects are perceived and to changes in the weighting of features during decision making (e.g., Goldstone, 1994; Goldstone, Lippa, \& Shiffrin, 2001; Johnson \& Mervis, 1997; Tanaka \& Curran, 2001). All of these computations (perceptual, semantic, and decision related) necessarily precede overt judgments about an object, even when factors such as stimulus masking or response deadlines are used to disrupt or hasten aspects of processing. Because knowledge effects at any of these stages (or a combination of more than one) could affect the nature of the final behavioral response, demonstrating that such effects can arise under speeded conditions or without overt task demands does not provide information about the precise stage(s) of processing at which knowledge effects arise. To do this requires measures that can tap into specific processing stages with more precision, such as event-related brain potentials (ERPs).

ERPs are small voltage changes in the electroencephalogram (EEG) that are synchronized in time with stimulus or response events, such as the onset of a word or line drawing on a computer screen. Components of the ERP have been identified as markers of specific cognitive operations, so that ERPs can provide a functionally specific measure of processing over time. Thus, ERPs offer the possibility of looking for individual and interactive effects of similarity and knowledge on multiple stages of processing in parallel. In particular, ERPs provide a means of more directly testing the claim (Lin \& Murphy, 1997; Luhmann et al., 2006) that background knowledge (e.g., about a feature's functional significance to an object) forms part of the representation of category information in semantic memory and that knowledge effects thus arise relatively automatically and implicitly during semantic access.

Semantic access is reflected in the ERP as changes in the amplitude of the N400, a negative-going component with a medio-central scalp distribution that appears 300 $500 \mathrm{msec}$ after the onset of a meaningful or potentially meaningful stimulus (e.g., Kutas \& Hillyard, 1980b). The N400 arises from brain activity in a network that includes bilateral medial and anterior temporal lobe areas and the superior temporal gyrus, which collectively are associated with the representation and access of meaning-related information (Nobre \& McCarthy, 1995; Tse et al., 2007). Accordingly, as has been reviewed by Kutas and Federmeier (2000), the N400 is elicited by all potentially meaningful stimuli, including not only words and word-like strings, but also environmental sounds, line drawings, and pictures. The N400 has been shown to be sensitive to a wide range of variables associated with the ease of retrieving information from semantic memory and integrating that information with context. For example, N400s are reduced for items that are repeated or contextually appropriate, as compared with items that are singly presented or contextually inappropriate. N400 amplitude has also been shown to be sensitive to semantic category structure, including typicality (e.g., Heinze, Münte, \& Kutas, 1998), and to the expected functions of common objects, with more negative responses to violations of semantic knowledge (e.g., attempting to use a rolling pin to shave one's face; Sitnikova, Kuperberg, \&
Holcomb, 2003). However, the N400 is insensitive to nonlinguistic manipulations of meaningful stimuli (e.g., improbable word font changes; Kutas \& Hillyard, 1980a) or to language-related violations that do not affect meaning (e.g., grammatical errors; Kutas \& Hillyard, 1983).

The functional specificity of the N400 makes it an ideal measure for testing claims about the representation of background knowledge in semantic memory and its use during object processing. Therefore, we conducted a pair of ERP experiments in which stimuli and paradigms derived from Lin and Murphy (1997) were used. Participants learned to a criterion level sets of novel object categories, including the physical form of the object and several key features, the object's name, and background knowledge about its function. At test, they were cued with a category name and then shown novel exemplars that had all of the learned features, were missing one feature (which was either essential or nonessential to the functioning of the object), or were missing two features. The participants either made a category membership judgment (for which background knowledge would likely be important; Experiment 1 ) or determined whether all the learned features were present (i.e., a parts detection judgment, for which background knowledge was incidental; Experiment 2).

For well-learned semantic categories, the N400 has been shown to be sensitive to similarity in a graded manner; that is, following a category name cue, exemplars that contain more category-relevant features elicit smaller N400s than do those containing fewer features (Heinze et al., 1998). Thus, if newly learned semantic category information is stored and processed similarly (to our knowledge, this is the first ERP experiment to address the processing of newly learned semantic categories), we would expect $\mathrm{N} 400$ responses in the present experiment to be graded with the number or novelty of features, with the most facilitation (smallest N400s) to exemplars containing all the learned features and the least facilitation (largest N400s) to exemplars missing two learned features. Of special interest, then, are the effects of knowledge, which would be expected to manifest as differences based on the importance of the features for object function. If the behavioral patterns seen in Lin and Murphy (1997) arise because information about the functional significance of the features is stored in semantic memory, we would expect to see more N400 facilitation for exemplars missing nonessential features (which are represented and processed more like complete exemplars) than for those missing essential features. A comparison of the pattern across the two experiments should further reveal whether knowledge-based effects on categorization depend on the nature of the task and, in particular, on whether the task makes explicit reference to that knowledge.

\section{EXPERIMENT 1}

In Experiment 1, we asked participants to determine whether or not novel exemplars would function as a member of a named category (i.e., to make a category membership judgment), in order to examine the effects of background knowledge on object processing under conditions 
in which participants are likely to make explicit reference to that knowledge. In particular, we examined whether the N400 component is sensitive to feature number, feature importance, or both.

In addition to effects that might emerge in relatively automatic stages of semantic processing, as indexed by the N400, effects due to attentionally driven aspects of semantic processing and memory retrieval could be seen later in the recording epoch, on the late positive complex (LPC; $500-900 \mathrm{msec}$ ). The LPC is a posterior positivity that follows the N400 and that has been linked to explicit recognition memory and controlled semantic processing (e.g., Olichney et al., 2000; Swaab, Brown, \& Hagoort, 1998). During the categorization of novel exemplars, LPC amplitude is graded by the frequency of individual features, with stimuli that contain more frequent features eliciting greater positivity (Folstein \& Van Petten, 2004). The LPC has also been shown to be sensitive to the degree of similarity between targets and distractors, suggesting that it indexes the difficulty of the classification judgment, rather than simple perceptual matching (e.g., Azizian, Freitas, Parvaz, \& Squires, 2006).

Finally, any effects on initial object perception would be seen prior to the N400. In particular, several frontal components (the frontal P2 and $\mathrm{N} 2 \mathrm{~b}$ ) have been found to be sensitive to aspects of higher level visual processing and attentional allocation (i.e., target detection and novelty detection, respectively; Daffner et al., 2000; Folstein \& Van Petten, 2008; Luck \& Hillyard, 1994) and may, therefore, reveal similarity or knowledge effects that arise during initial perceptual assessment.

\section{Method \\ Participants \\ Twenty-four University of Illinois undergraduates (12 men and 12 women; ages, 18-30 years; average age, 20 years) participated in this study for class credit. The participants were right-handed native English speakers who had not been exposed to another language early in their childhood and did not have vision problems.}

\section{Materials}

The materials for this study were modeled after those used by Lin and Murphy (1997). In total, there were 18 different novel object categories ( 8 taken from the original set used by Lin and Murphy and 10 more created for this study), all of which were presented to the participants as artifacts used in the make-believe country of "Quine." Training stimuli consisted of line drawings paired with a novel category name and a written description of the object category. Three prototypical examples of each category, one of which had its parts labeled, were printed together on the same page (as in Figure 1). Category names were pronounceable pseudowords (e.g., lurt, prabe, batin) four to six characters in length. Descriptions of the novel artifacts provided information about each object's purpose and pointed out the three labeled features of each, explaining how those features aided the object's function. Two of the features were essential to the proper functioning of the object, and one feature was useful but nonessential. Each artifact was paired with two different descriptions, in which the functional significance of the features was alternated. In the alpha description, Features 1 and 3 were essential but Feature 2 was nonessential, whereas in the beta description, Features 2 and 3 were essential but Feature 1 was nonessential (see Figure 1 for an example of the two different descriptions). Each participant read only one description of the object, and descriptions were counterbalanced across the experiment. Features $(1,2$, and 3$)$ were mentioned in randomized orders in the descriptions, although these orders were consistent across the alpha and beta versions of each object.

There were four types of test stimuli (Figure 2). Complete (COM) exemplars were novel instances of the object category that contained all of the described features $(1,2$, and 3$)$. Two types of test items were missing one of the described features (missing-one-feature [M-ONE] exemplars). In the missing-essential-feature (M-ESS) exemplars, one of the described features that was essential to object functioning (Feature 1 in the alpha description and Feature 3 in the beta description) was deleted so that the object was no longer fully functional. In the missing-nonessential-feature (M-NON) exemplars, the nonessential feature (Feature 2 in the alpha description and Feature 1 in the beta description) was deleted. For their fourth condition, Lin and Murphy (1997) used control exemplars that were missing both an essential and a nonessential feature. However, with only one visual feature remaining, these exemplars were notably simplified, relative to the other exemplar types. In order to have a control condition that was more similar in perceptual complexity to the other exemplar types, our missing-two-feature (M-TWO) exemplars were constructed by altering or replacing (rather than removing) both Features 1 and 3, so that the object would not be fully functional under either description but would have a number of features similar to that for the other exemplar types. Two test items of each type (COM, M-ESS, M-NON, and M-TWO) were created for each of the 18 object categories (144 total). The 18 categories were divided into three blocks, with 6 categories (48 test items) in each block.

\section{Experimental Procedure}

Following instructions, the participants were directed to consider a situation in which they needed to loan a real object (a broom) to a friend. They were shown several perceptually similar objects that were either complete or missing a subset of features and were asked to decide which ones they would lend to their friend. The ex-
A

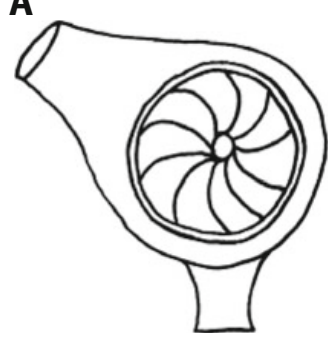

C

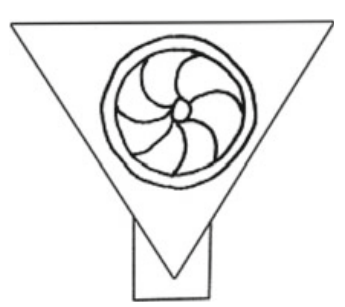

B

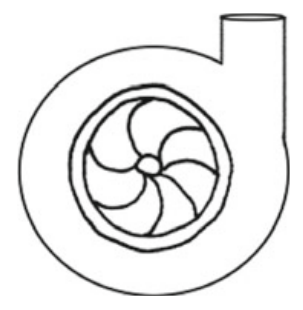

D

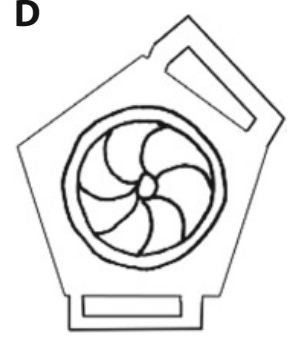

Figure 2. Example of test stimuli. Four types of test stimuli were used, as exemplified here: complete items (A) that contained all the labeled features from the training set, items that were missing one learned feature $(B$ and $C$ ), and items in which two features were missing and/or altered (D). Items $B$ and $C$ are further differentiated into missing-essential-feature $(C$ for the alpha description and $B$ for the beta description) and missingnonessential-feature ( $B$ for the alpha description and $C$ for the beta description) conditions. No stimuli from the training set were also used as test items. 
perimenter provided feedback, emphasizing that even incomplete objects could be loaned, provided that they were still able to function as a broom. The participants then completed a practice block using real-world objects, with COM, M-ESS, M-NON, and M-TWO exemplars. As in the experimental task, the participants were asked to respond yes if they felt that the exemplar was a member of the named category and no if it was not.

Once they had completed the practice block, the participants were given the first set of six object categories to learn. Each participant was randomly assigned to learn either the alpha or the beta descriptions, and the order of the three study-test blocks was counterbalanced across participants. When the participant indicated that he or she was ready, learning was assessed by showing the participant a complete exemplar from each category and asking him or her to name the object, describe its function, and point out and describe each of its features. If the participant was unable to complete the test successfully, he or she was asked to look over the items again and was retested until he or she could remember the names and functions perfectly. At that point, the test block for those items commenced.

During both practice and experimental blocks, the participants were seated in a quiet testing room $100 \mathrm{~cm}$ away from a computer screen and were asked to respond as quickly and accurately as possible (by pressing one of two buttons, held in each hand), pressing yes if they felt that the exemplar was a member of the named category and no if it was not. Hand used to respond yes was counterbalanced across participants. The stimuli were presented in one of six different randomized orders, also counterbalanced across participants. The participants were told to try to avoid eye movements or blinks while the test items were on the screen. On each trial, a category name appeared for $1,500 \mathrm{msec}$ in the center of the screen, followed by a 1-sec interstimulus interval. An exemplar was then presented in the center of the screen and remained on the screen until the participants registered a response. A 3,000-msec blank screen separated the trials.

The participants learned and were tested on the three sets of six categories in succession. They were explicitly told that items from earlier learning sets would never reappear in subsequent test blocks. Each block lasted approximately $7 \mathrm{~min}$, and the entire experimental session (including setup and the three learning and test sessions) lasted approximately $2 \mathrm{~h}$.

\section{EEG Recording, Data Processing, and Analyses}

Electroencephalographic (EEG) activity was recorded from 26 geodesically arranged $\mathrm{Ag} / \mathrm{AgCl}$ electrodes embedded in an electrode cap (see Figure 3 for a schematic of the electrode locations), and an electrode placed below the left eye monitored blinks. These channels were referenced to the left mastoid online and then rereferenced offline to the average of the left and right mastoid. In addition, horizontal eye movements were monitored via the electrooculogram (EOG), collected from a bipolar montage of electrodes placed at the outer canthus of each eye. Impedances for all electrodes were kept below $5 \mathrm{k} \Omega$. EEG and EOG were sampled at $250 \mathrm{~Hz}$, band-pass filtered from 0.02 to $100 \mathrm{~Hz}$, and amplified with a gain of 10,000 using a Sensorium amplifier.

Prior to averaging, trials containing eye movements or other artifacts were rejected, with thresholds individualized to each participant. Blinks were also detected and were corrected for 4 participants, using a procedure developed by Dale (1994); the remaining 20 participants did not have enough blinks to obtain a stable filter. Across participants, an average of $7 \%$ of the critical trials were lost due to artifacts. ERPs were computed from $100 \mathrm{msec}$ before the onset of critical words to $920 \mathrm{msec}$ after. Averages of artifact-free ERPs were calculated for each condition after subtraction of the $100-\mathrm{msec}$ prestimulus baseline. Measurements were taken after a digital band-pass filter of $0.2-20 \mathrm{~Hz}$ was applied (with the exception of peak latency measurements, which were band-pass filtered from 0.2 to $5 \mathrm{~Hz}$ ).

Electrophysiological markers of featural similarity processing were examined via comparisons among the COM, M-ONE, and M-TWO conditions, and electrophysiological markers of knowledge-

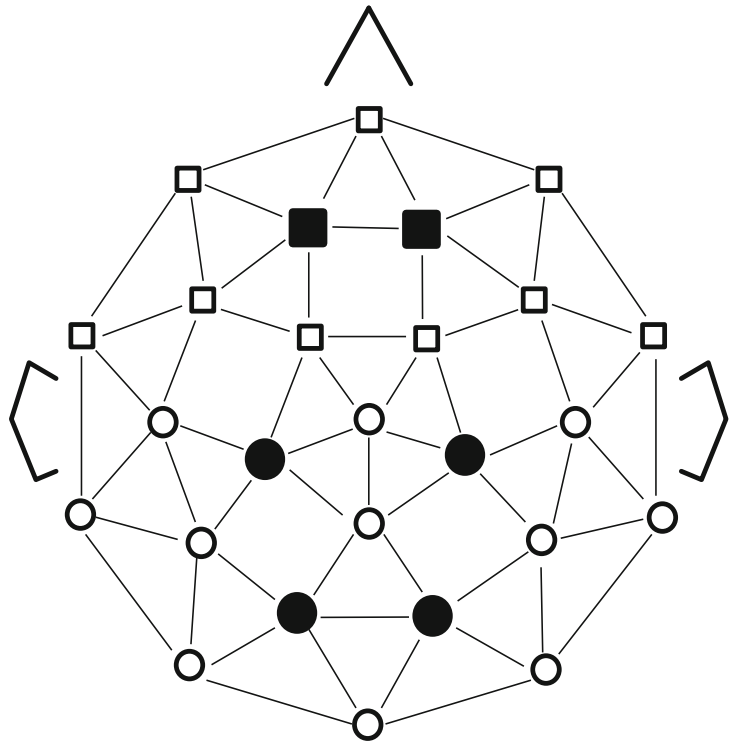

Figure 3. Locations of the 26 scalp electrodes used in the experiments (circles and squares on the figure). Each square represents an electrode used in the frontal channel analyses (used for P2/ N2b), and each circle represents an electrode used for centroposterior channel analyses (used for N400 and LPC). Filled-in shapes show the locations of the channels shown in Figures 4, 6, 7 , and 8.

based effects (sensitivity to the functional significance of features) were assessed by comparisons between the M-ESS and M-NON conditions. Analyses were focused on the N400 component, measured from 300 to $500 \mathrm{msec}$ over the 15 posterior electrodes where this effect is most prominent (Figure 3). Additional analyses were performed on the LPC, measured from 500 to $900 \mathrm{msec}$ across the same 15 posterior electrodes as those used in the N400 analysis. The frontal P2/N2b was measured from 150 to $250 \mathrm{msec}$ over 11 frontal sites that typically show such effects. Given the similar time course, distribution, and direction of the P2 and the N2b (with decreased feature overlap, the N2b tends to be more negative and the P2 less positive), these effects may be difficult to dissociate; for present purposes, however, the $\mathrm{P} 2$ and $\mathrm{N} 2 \mathrm{~b}$ can jointly be considered indices of effects at perceptual and attentional levels of processing. For all ERP analyses, the reported $p$ values reflect the Huynh-Feldt correction for repeated measures with more than one degree of freedom. Interactions with electrode site will be reported only when of theoretical significance.

\section{Behavior}

\section{Results}

Response choice. Endorsement rates (the percentage of yes responses, indicating that an exemplar was believed to be a member of the named category) are presented in Table 1. Endorsement rates were graded by feature number: highest for COM exemplars, lowest for M-TWO exemplars, and intermediate for the two M-ONE exemplars, as confirmed in an ANOVA with four levels of exemplar type $[F(3,69)=208.79, p<.0001, \varepsilon=.488]$. For items missing a single feature, the participants were significantly more likely to endorse M-NON than M-ESS exemplars. Table 2 shows the full set of pairwise comparisons across exemplar types. Overall, the participants were more likely 
Table 1

Endorsement Rates and Response Times (RTs), Experiment 1

\begin{tabular}{|c|c|c|c|c|c|}
\hline \multirow[b]{2}{*}{ Condition } & \multicolumn{2}{|c|}{ Endorsement (\%) } & \multicolumn{2}{|c|}{ RT (msec) } & \multirow[b]{2}{*}{ Trial Counts } \\
\hline & $M$ & $\overline{S E}$ & $M$ & $S E$ & \\
\hline $\mathrm{COM}$ & 95.9 & 0.7 & 1,662 & 77 & $33.8(23-36)$ \\
\hline M-NON & 37.2 & 5.5 & 1,916 & 116 & $33.9(21-36)$ \\
\hline M-ESS & 11.3 & 1.6 & 1,727 & 95 & $33.5(23-36)$ \\
\hline M-TWO & 11.0 & 2.4 & 2,298 & 162 & $33.0(23-36)$ \\
\hline
\end{tabular}

Note-Trial counts report mean numbers of trials used in ERP analyses and, therefore, include both yes and no responses; the ranges for individual participants are reported in parentheses.

Table 2

Pairwise Comparisons $[F(1,23)]$ of Behavioral Measures, Experiment 1

\begin{tabular}{|c|c|c|c|c|c|}
\hline \multirow[b]{2}{*}{ Condition } & \multicolumn{4}{|c|}{ Condition } & \multirow[b]{3}{*}{ Endorsement rate comparisons } \\
\hline & M-TWO & M-ESS & M-NON & COM & \\
\hline $\mathrm{COM}$ & $1,317.63^{* * *}$ & $2,584.91^{* * *}$ & $122.84^{* * * *}$ & - & \\
\hline M-NON & $24.75^{* * *}$ & $27.12^{* * *}$ & - & $7.54^{*}$ & \\
\hline M-ESS & n.s. & - & $7.31^{*}$ & n.s. & Response time comparisons \\
\hline M-TWO & - & $37.97^{* * *}$ & $13.96^{* *}$ & $21.52^{* * *}$ & \\
\hline
\end{tabular}

Note-White cells (upper left) present $F$ values and significance levels for all accuracy contrasts; gray cells (lower right) present $F$ values and significance levels for all response time contrasts. ${ }^{*} p<.05$. ${ }^{* *} p<$ $.01{ }^{* * *} p<.001$. n.s., not significant.

to treat exemplars missing functionally nonessential features as category members.

Response times. The median response times for each exemplar type are presented in Table $1 .{ }^{1}$ An ANOVA with four levels of exemplar type confirmed that the median time to execute a response differed across conditions $[F(3,69)=16.74, p<.0001, \varepsilon=.761]$. Response times were shortest to COM exemplars (largely associated with a yes response) and longest to M-TWO exemplars (largely associated with a no response). In addition, pairwise comparisons (Table 2) revealed lower response times to M-ESS than to M-NON exemplars. Thus, the participants judged the category membership of items missing essential features more quickly than they judged membership of items missing nonessential features. Overall, the behavioral results replicated Lin and Murphy (1997) in suggesting that speeded category membership judgments are influenced both by the number of expected features a category exemplar contains and by the functional significance of object features.

\section{ERPs}

Featural similarity. The traditional conceptualization of object categorization as attribute matching was tested by comparing the electrophysiological response to visual objects containing all or only some of the learned features. The following analyses will compare exemplars with all three learned features $(\mathrm{COM})$ with exemplars missing either one (M-ONE) or two (M-TWO) critical features. The M-ONE analysis is collapsed across feature importance, which will be examined separately in the following section.

Figure 4 shows the ERP responses to COM, M-ONE, and M-TWO exemplars at six channels across the scalp. Condition-related differences appear to begin over frontal sites around $200 \mathrm{msec}$, in the form of increased negativ- ity to the M-TWO exemplars, as compared with the other conditions $(\mathrm{P} 2 / \mathrm{N} 2 \mathrm{~b})$. A graded response to the conditions is evident after this, with the most negativity to M-TWO exemplars and the most positivity to COM exemplars in the period encompassing the N400 (300-500 msec) and LPC (500-900 msec).

Frontal P2/N2b (150-250 msec). The earliest effect of number of learned features emerged over frontal sites

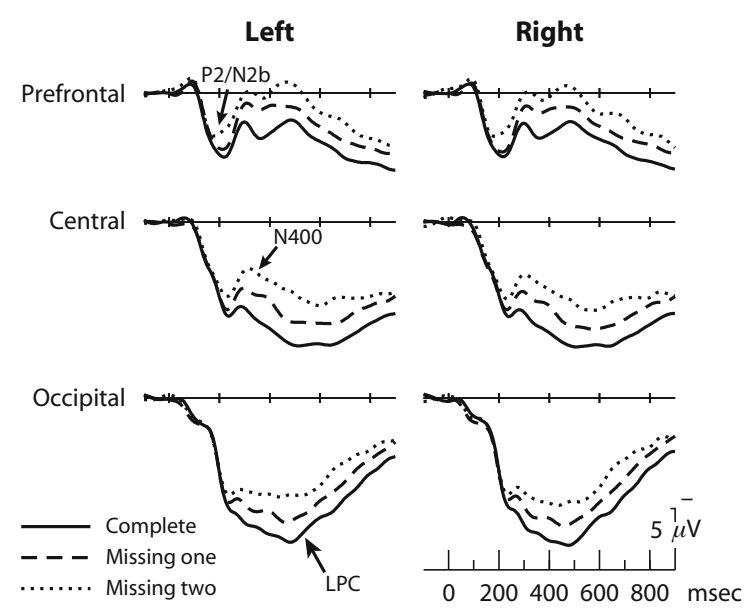

Figure 4. ERP effects of feature number in Experiment 1. Plotted are ERP responses to COM, M-ONE, and M-TWO exemplars at six representative channels (filled-in shapes in Figure 3). Negative is plotted up here and in all the subsequent figures. In the figure, solid lines represent complete exemplars, dashed lines represent missing-one-feature exemplars, and dotted lines represent missing-two-features exemplars. Arrows point to the three main similarity-based effects: a frontal P2/N2b, which distinguished between M-TWO and other exemplar types, and a centro-posterior $\mathrm{N400}$ and LPC, both of which showed graded responses to the conditions (COM $>$ M-ONE $>$ M-TWO). 
between 150 and $250 \mathrm{msec}$, with increased negativity to M-TWO exemplars, relative to M-ONE and COM exemplars. An ANOVA with 3 levels of exemplar type (COM, M-ONE, and M-TWO) and 11 levels of electrode site (frontal channels marked in Figure 3) confirmed the effect of exemplar type $[F(2,46)=5.81, p<.01, \varepsilon=.857]$. Responses were more negative to M-TWO exemplars than to M-ONE exemplars [3.1 vs. $4.0 \mu \mathrm{V} ; F(1,23)=10.23$, $p<.01]$, which, in turn, did not differ from responses to COM exemplars $[4.3 \mu \mathrm{V} ; F(1,23)=0.66$, n.s.].

N400 (300-500 msec). A widespread negativity with a peak latency (measured for the difference between M-TWO and COM exemplars at a midline parietal channel) of $427 \mathrm{msec}$ appeared to be graded by the number of learned features that a novel exemplar contained. This negativity had a medial-central focus, characteristic of the N400, as confirmed by a distributional analysis conducted on the difference wave (a point-by-point subtraction of M-TWO minus COM), using the 16 scalp sites that could be divided into factors of hemisphere (left vs. right), laterality (lateral vs. medial), and anteriority (prefrontal, frontal, central/parietal, or occipital), which revealed a significant main effect of laterality $[F(1,23)=$ $46.90, p<.0001]$ modulated by an interaction between laterality and anteriority $[F(3,69)=2.74, p=.05$, $\varepsilon=.850]$. Figure $5 \mathrm{~A}$ shows a topographic map of the distribution.

On the basis of the distributional analysis, conditionrelated effects on the N400 were examined over central and posterior sites, using an ANOVA with 3 levels of exemplar type (COM, M-ONE, or M-TWO) and 15 levels of electrode site (central/posterior channels marked in Figure 3). As is visible in Figure 4, the N400 was graded by feature number $[F(2,46)=31.98, p<.0001, \varepsilon=$ .838], with M-TWO exemplars being more negative than M-ONE exemplars [5.5 vs. $6.9 \mu \mathrm{V} ; F(1,23)=25.62, p<$ $.0001]$, which, in turn, were more negative than COM exemplars $[8.3 \mu \mathrm{V} ; F(1,23)=18.13, p<.001]$.

Late positive complex $(500-900 \mathrm{msec})$. The graded pattern of response to the three exemplar types continued beyond the N400 into the late part of the epoch. This was confirmed in an ANOVA of exemplar type (COM, M-ONE, or M-TWO) and electrode site (15 central/ posterior channels marked in Figure 3), which revealed a main effect of exemplar type $[F(2,46)=14.38, p<.001$, $\varepsilon=.845]$, with more negative responses to M-TWO than to M-ONE exemplars [4.7 vs. $5.7 \mu \mathrm{V} ; F(1,23)=10.07$, $p<.01]$ and more negative responses to M-ONE than to COM exemplars $[6.8 \mu \mathrm{V} ; F(1,23)=9.19, p<.01]$.

Summary. Consistent with predictions based on data seen for well-learned semantic categories, the N400 to newly learned category exemplars was graded by featural similarity, so that responses were most positive (facilitated) to novel exemplars with all the learned features (COM), intermediate in amplitude to exemplars missing a single feature (M-ONE), and most negative to exemplars with two features that had been altered or replaced (M-TWO). This graded pattern continued into later parts of the epoch (LPC), associated with more explicit aspects of processing. Finally, the presence of novel featural information in the M-TWO condition was associated with an early ERP signature, in the form of a decreased frontal P2 and/or en-

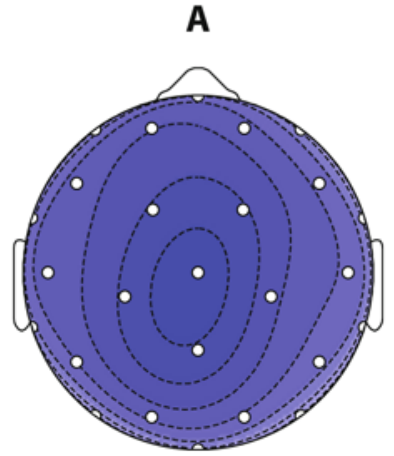

Experiment 1 N400 Effect

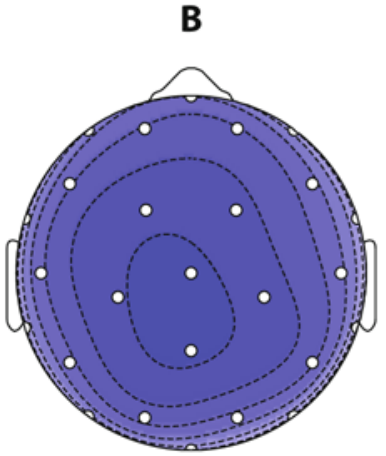

Experiment 2 N400 Effect

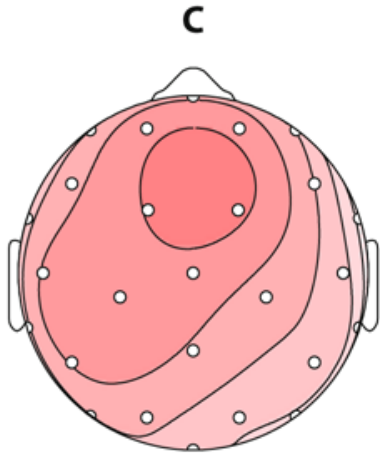

Experiment 1

Featural

Importance Effect

Figure 5. Topographic maps of effect distributions, 350-450 msec. Voltage maps of effect distributions for Experiments 1 and 2 are shown from a top view of the head. Shading represents mean voltages in a 100-msec time window centered around $400 \mathrm{msec}(350-450 \mathrm{msec}$ ). The $\mathrm{N} 400$ effect was calculated by subtracting COM waveforms from M-TWO waveforms, and the featural importance effect was calculated by subtracting M-ESS waveforms from M-NON waveforms. (Thus, in both cases, more-expected items were subtracted from less-expected items.) (A) The N400 effect from Experiment 1 . (B) The N400 effect from Experiment 2. (C) The featural importance effect from Experiment 1. The N400 effect in both experiments had a distribution typical of this component, with a maximum over medial, centro-posterior electrode sites. In contrast, the featural importance effect was positive- rather than negative-going and had a more frontal focus. 
hanced N2b, suggesting that perceptual and/or attentional systems were engaged differently in this condition.

Featural importance. Next, a set of analyses were done on the M-ONE exemplars, comparing those missing a feature essential to object function (M-ESS) with exemplars missing a nonessential feature (M-NON). Figure 6 overlaps the ERP responses to M-ESS and M-NON exemplars at six electrode sites. If the functional significance of features is encoded in semantic memory, we should expect to see reduced N400s to exemplars missing nonessential features (M-NON). However, this pattern did not occur. Instead, these conditions diverged with a focus over prefrontal and frontal sites, beginning around $300 \mathrm{msec}$, with M-ESS exemplars eliciting more positive responses than did M-NON exemplars. Analyses (including all channels) were done between 300 and $500 \mathrm{msec}[\mathrm{M}-\mathrm{ESS}=5.22 \mu \mathrm{V}$, $\mathrm{M}-\mathrm{NON}=3.93 \mu \mathrm{V} ; F(1,23)=8.13, p<.01]$ and between 500 and $900 \mathrm{msec}[F(1,23)=7.15, p<.05]$.

As can be seen in Figure $5 \mathrm{C}$, in addition to being opposite in direction from the N400 (i.e., with the more expected condition yielding more negative responses than did the less expected condition), this featural importance effect has a different distribution from the temporally overlapping N400 effect observed for number of features. A distributional ANOVA (again using 16 electrode sites divided into factors of hemisphere, laterality, and anteriority) comparing the N400 difference wave (point-by-point subtraction of COM from M-TWO) with the featural importance difference wave (M-NON - M-ESS) between 300 and $500 \mathrm{msec}$ revealed an interaction of effect type $X$ laterality $\times$ anteriority $[F(3,69)=4.21, p<.02, \varepsilon=$ $.807]$. Over medial sites where both effects tend to be larger, the functional significance effect has a more fron-
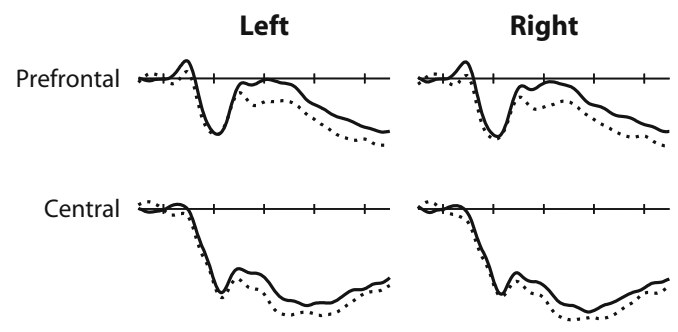

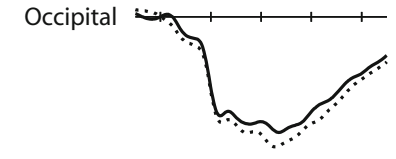

- Missing one, nonessential ....... Missing one, essential
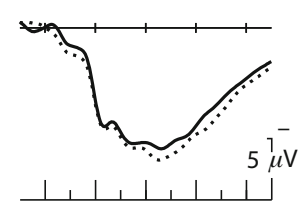

$0200400600800 \mathrm{msec}$
Figure 6. ERP effects of featural importance for Experiment 1. ERP responses to $M-E S S$ and $M-N O N$ exemplars at six representative channels (filled-in shapes in Figure 3). Solid lines represent exemplars missing a feature that is nonessential to object function (M-NON), and dotted lines represent exemplars missing an essential feature (M-ESS). Particularly over frontal electrode sites, M-ESS exemplars elicited enhanced positivity, relative to M-NON exemplars, beginning around $300 \mathrm{msec}$ and continuing throughout the recording epoch. tal distribution, being biggest over prefrontal and frontal electrode sites.

Thus, knowledge-based effects could be seen in the ERP signal beginning around $300 \mathrm{msec}$ after stimulus onset and continuing throughout the epoch. ${ }^{2}$ Although this effect overlapped in time with the N400 (and LPC) response to featural similarity, differences in the directionality (more positive to M-ESS than to M-NON) and distribution (more frontal for the feature importance difference) of the effects show that they are dissociable responses.

\section{Discussion}

The goal of Experiment 1 was to examine how featural similarity and background knowledge are used during judgments about novel category exemplars and, in particular, to test the hypothesis that knowledge effects in behavior arise because information about the functional significance of features is encoded in semantic memory and, therefore, used automatically during object processing. Clear effects of similarity were evident in the ERP responses to new exemplars of recently learned categories from fairly early in processing. Exemplars with relatively novel featural information (M-TWO exemplars, in which two learned features had been altered or replaced) were differentiated from other exemplar types within the first $200 \mathrm{msec}$ of stimulus apprehension. The timing and distribution of this difference are consistent with frontal P2 and/or N2b effects, which are believed to reflect aspects of target detection and novelty detection, respectively. In the present context, it would be difficult to dissociate the two components (although at least one prior study has suggested that the N2b effect may be unlikely to arise, except under more attentionally demanding conditions than those imposed by the present tasks; Folstein, Van Petten, \& Rose, 2008). However, the effect clearly indicates that the M-TWO test items are sufficiently perceptually dissimilar from the training exemplars (either within the cued category or across the set overall) to elicit distinct brain responses during early stages of object processing.

Following this early effect, a fully graded response to the number of expected features presented (with differences between M-ONE and COM exemplars, as well as between M-TWO and M-ONE exemplars) was observed beginning around $300 \mathrm{msec}$ after stimulus onset. This response took the form of a negativity with a peak amplitude just after $400 \mathrm{msec}$ and a widespread distribution with a medio-central focus. The functional sensitivity of this response, with reduced negativity to more expected exemplar types, and its timing and scalp distribution are consistent with its characterization as an N400. The N400 has been strongly linked to relatively implicit aspects of semantic access and integration (e.g., Kutas \& Federmeier, 2000; Olichney et al., 2000). The fact that the N400 was graded by the number of features a test exemplar shared with the training set suggests that, for newly learned categories as for more established ones, assessments of featural similarity form an integral part of how semantic representations of objects are accessed. This graded effect continued into the LPC time window, 
associated with more explicit and attentionally controlled aspects of semantic processing.

Knowledge-based effects were evident in the participants' behavioral responses and the corresponding ERP recordings. Replicating Lin and Murphy (1997), the results showed that the participants were faster and more likely to reject as category members exemplars that were missing features essential to object function, as compared with exemplars that were missing nonessential features. If these effects arise because the functional significance of object features is encoded in semantic memory and automatically accessed during object processing, we would expect to see knowledge effects on the N400, co-occurring with the effects of feature number just described. In particular, such a view predicts that exemplars missing nonessential features (M-NON) would be treated more like complete exemplars than would those missing essential features (M-ESS) and, thus, that M-NON exemplars would elicit more reduced (positive) N400 responses. However, effects of knowledge emerged instead in the form of more negative frontal potentials to M-NON than to M-ESS exemplars, beginning around $300 \mathrm{msec}$ and continuing until late parts of the epoch. This featural importance effect overlapped in time and over some electrode sites with the featural similarity effect on the N400 (and LPC) but differed in both direction (for the N400, more positive to items that were more likely to be called category members; for the featural importance effect, more positive to items that were less likely to be called category members) and distribution (with a central-posterior maximum for the N400 but a more anterior focus for the featural importance effect). Thus, although both background knowledge and similarity can influence object processing from a similar, early point in time, these effects appear to be dissociable, and, critically, knowledge effects do not seem to arise from the kind of semantic access and integration processes that have long been associated with the N400.

If the featural importance effect is not an N400, what kind of effect might it be? Because the present study is novel in many ways with respect to the current ERP literature, it is difficult to make definitive links to other components. An ERP response with some similar properties has been described in studies of object processing (Schendan $\&$ Kutas, 2002, 2003, 2007). Like the featural importance effect, this $N 350$ effect begins around $300 \mathrm{msec}$ (peaking shortly thereafter), can be fairly sustained, and has a fronto-central distribution. The N350 is sensitive to the ease of object processing, being smaller (i.e., more positive) to objects that are correctly identified than to those that are not (Schendan \& Kutas, 2002), to objects presented in canonical as opposed to noncanonical views, and to repeated objects (Schendan \& Kutas, 2003, 2007). The featural importance effect observed here shows a directionality similar to that of these N350 effects, being more positive for the M-ESS items, which also seemed to be easier to classify both in Lin and Murphy (1997) and in the present experiment, given the more rapid classification judgments for M-ESS than for M-NON items.

Studies of object classification (Azizian et al., 2006; Folstein \& Van Petten, 2004; Folstein et al., 2008) have also observed a fronto-central negativity (200-600 msec), which seems to arise when different aspects of a stimulus prime opposing responses. This effect has been linked to the N2, an ERP component that has been associated with response conflict (e.g., Nieuwenhuis, Yeung, van den Wildenberg, \& Ridderinkhof, 2003; Pfefferbaum, Ford, Weller, \& Kopell, 1985). Our effect could be related to response conflict if the M-NON items prime both a rejection response (due to their missing feature) and an endorsement response (due to knowledge that the missing feature is not essential to function).

Because Experiment 1 asked the participants to make overt judgments about category membership, it is likely that background knowledge was used explicitly in service of the task. Given evidence that knowledge effects can arise even when knowledge is not called upon by the task (e.g., Lin \& Murphy, 1997), it is of interest to determine whether the ERP featural importance effect reflects a process that occurs during any task or whether it manifests only in tasks that, like that in Experiment 1, direct participants to explicitly consider feature importance in making their judgments about the objects. N350 repetition effects have been seen even during indirect memory tasks (Schendan \& Kutas, 2003, 2007), suggesting that they can sometimes occur under implicit conditions; if the featural importance effect we observed here is an N350 or something similar, it is thus possible that we would see knowledge effects on the ERP even in a task that does not explicitly require participants to consider object function. On the other hand, N2 effects would be expected to occur only under task conditions in which knowledge of feature importance led to response conflict. Therefore, in Experiment 2, a parts detection task was used (as in Lin \& Murphy, 1997) to examine the influence of similarity and featural importance on processing when assessments of category membership and function were incidental to participants' decisions.

\section{EXPERIMENT 2}

In Experiment 2, we asked participants to indicate whether novel category exemplars did or did not contain all of their learned parts in order to examine the effects of background knowledge on object processing under conditions in which the participants were not likely to make explicit reference to that information. Experiment 1 identified a frontally distributed ERP correlate of feature importance; we were particularly interested in testing the ubiquity of this effect across different task demands. Again, we examined not only knowledge effects, but also similarity effects, with emphasis on the N400, LPC, and $\mathrm{P} 2 / \mathrm{N} 2 \mathrm{~b}$.

\section{Method}

\section{Participants}

Twenty-four University of Illinois undergraduates (12 men and 12 women; ages, $18-30$ years; mean age, 19.6 years) participated in the study for class credit. None of these participants had previously been in Experiment 1. The participants had normal vision and were right-handed native English speakers who had not been exposed to another language early in life. 


\section{Materials and Procedure}

The same materials and study phase procedures as those in Experiment 1 were used in Experiment 2. At test, however, the participants were asked to perform a parts detection task, by responding yes only to those objects that contained all of the studied features and responding no to an object with any feature(s) missing.

\section{EEG Recording, Data Processing, and Analyses}

The recording parameters and data processing and analysis procedures used in Experiment 2 were the same as those in Experiment 1. Two of the participants were blink corrected using the procedure developed by Dale (1994). On average, $16 \%$ of the critical trials were lost due to artifacts.

\section{Results}

\section{Behavior}

Accuracy. Correct responses in the parts detection task were yes responses to COM items and no responses to all other item types; accuracy across conditions is reported in Table 3. An omnibus ANOVA with four levels of exemplar type revealed a graded effect of exemplar type $[F(3,69)=$ 13.18, $p<.0001, \varepsilon=.899$ ], with performance being best for COM exemplars, worst for M-TWO exemplars, and intermediate for the two types of M-ONE exemplars. Critically, replicating Lin and Murphy (1997), responses were significantly more accurate for M-ESS than for M-NON exemplars (pairwise comparisons are shown in Table 4). The participants were thus better able to identify exemplars missing a single feature as incomplete when that missing feature was essential to the object's function, revealing an influence of the functional significance of the features on performance.

Response times. Median time to execute a correct response (given in Table 3) was analyzed across conditions, using an ANOVA with four levels of exemplar type, revealing a significant effect $[F(3,69)=27.83, p<.0001, \varepsilon=$ $.644]$. Pairwise comparisons (shown in Table 4) revealed that responses were slower for M-TWO exemplars than for the other exemplar types and, in line with the accuracy results, were slower to M-NON than to M-ESS exemplars. Thus, in this experiment (as in Experiment 1, although not in Lin \& Murphy, 1997), knowledge of a missing feature's importance also affected response speed.

\section{ERPs}

Featural similarity. As in Experiment 1, featural similarity was examined by contrasting complete exemplars (COM) with those missing either one (M-ONE) or two (M-TWO) learned features. Figure 7 shows the ERP responses to COM, M-ONE, and M-TWO exemplars at six channels across the scalp. Condition-related differences again first emerged around $200 \mathrm{msec}$ over frontal sites, with greater negativity $(\mathrm{P} 2 / \mathrm{N} 2 \mathrm{~b})$ to the M-TWO exemplars, as compared with the other conditions. Following the sensory components, a graded response to the conditions is evident on the N400 (300-500 msec) and the LPC (500-900 msec). Effects were analyzed in these three time windows, using only ERPs associated with correct responses (i.e., yes for COM exemplars, and no for M-ONE and M-TWO exemplars).

Frontal P2/N2b (150-250 msec). Mean amplitude responses between 150 and $250 \mathrm{msec}$ were analyzed using an ANOVA with 3 levels of exemplar type (COM, M-ONE, and M-TWO) and 11 levels of electrode site (frontal channels shown in Figure 3), which revealed that P2/N2b amplitude was sensitive to feature number [exemplar type: $F(2,46)=$ $6.99, p<.01, \varepsilon=.923]$. Replicating the pattern seen in Experiment 1 , responses were more negative to M-TWO exemplars than to M-ONE exemplars $[2.1$ vs. $3.2 \mu \mathrm{V} ; F(1,23)=$ $12.17, p<.01]$, which did not differ from responses to COM exemplars $[3.2 \mu \mathrm{V} ; F(1,23)=0.03$, n.s. $]$.

N400 (300-500 msec). Peak latency of the N400 was $444 \mathrm{msec}$ over the midline parietal channel. The distribu-

Table 3

Endorsement Rates and Response Times (RTs), Experiment 2

\begin{tabular}{|c|c|c|c|c|c|}
\hline \multirow[b]{2}{*}{ Condition } & \multicolumn{2}{|c|}{$\begin{array}{l}\text { Accuracy } \\
\text { (\% Correct) }\end{array}$} & \multicolumn{2}{|c|}{ RT (msec) } & \multirow[b]{2}{*}{ Trial Counts } \\
\hline & $M$ & $S E$ & $M$ & $S E$ & \\
\hline COM & 95.6 & 0.9 & 1,620 & 91 & $28.83(22-36)$ \\
\hline M-NON & 86.8 & 1.2 & 1,599 & 78 & $26.88(20-34)$ \\
\hline M-ESS & 91.8 & 1.2 & 1,502 & 76 & $28.63(23-34)$ \\
\hline M-TWO & 86.3 & 2.0 & 2,180 & 148 & $26.33(17-34)$ \\
\hline
\end{tabular}

Note-Trial counts report mean numbers of trials used in ERP analyses and, therefore, include only correct trials (i.e., yes to COM exemplars, no to all others); ranges for individual participants are reported in parentheses.

Table 4

Pairwise Comparisons $[F(1,23)]$ of Behavioral Measures, Experiment 2

\begin{tabular}{lcccc}
\hline & \multicolumn{5}{c}{ Condition } \\
\cline { 2 - 5 } Condition & M-TWO & M-ESS & M-NON & COM \\
\hline COM & $18.37^{* * *}$ & $4.89^{*}$ & $35.22^{* * *}$ & - \\
M-NON & n.s. & $17.38^{* * *}$ & - & n.s. \\
M-ESS & $10.13^{* *}$ & - & $5.66^{*}$ & $3.12^{\dagger}$ \\
M-TWO & - & $43.89^{* * *}$ & $39.99^{* *}$ & $25.10^{* * *}$
\end{tabular}

Note-White cells (upper left) present $F$ values and significance levels for all accuracy contrasts; gray cells (lower right) present $F$ values and significance levels for all response time contrasts. ${ }^{*} p<.05 .{ }^{* *} p<.01 .{ }^{* * *} p<.001 . \quad{ }^{\dagger} p<.10$. n.s., not significant. 

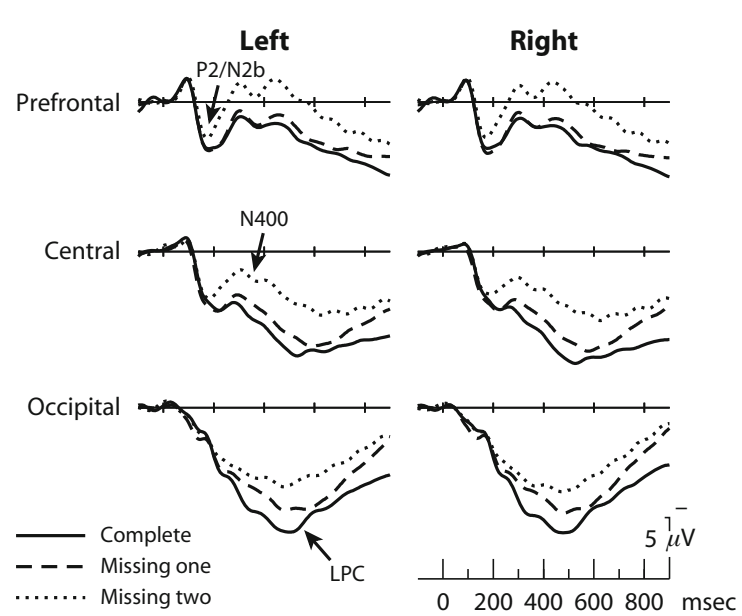

Figure 7. ERP effects of feature number for Experiment 2. ERP responses to COM, M-ONE, and M-TWO exemplars at six representative channels (filled-in shapes in Figure 3). Solid lines represent complete exemplars, dashed lines represent missing-onefeature exemplars, and dotted lines represent missing-two-features exemplars. Arrows point to the three main similarity-based effects, which replicated those in Experiment 1 (cf. Figure 4): a frontal P2/ N2b, and a centro-posterior N400 and LPC.

tion of the effect (see Figure 5B) was characterized on the difference wave (M-TWO minus COM) using 16 scalp sites divided into factors of hemisphere (left vs. right), laterality (lateral vs. medial), and anteriority (prefrontal, frontal, central/parietal, or occipital). Replicating the pattern seen in Experiment 1, there was a main effect of laterality $[F(1,23)=35.90, p<.0001]$, modulated by an interaction between laterality and anteriority $[F(3,69)=$ $10.12, p<.001, \varepsilon=.756]$, indicating an effect with a medio-central focus, as characteristic of the N400.

An ANOVA with 3 levels of exemplar type (COM, M-ONE, or M-TWO) and 15 levels of electrode site (central/posterior channels shown in Figure 3) revealed a main effect of exemplar type $[F(2,46)=27.08, p<.0001$, $\varepsilon=.936]$. M-TWO exemplars were more negative than M-ONE exemplars [3.8 vs. $5.3 \mu \mathrm{V} ; F(1,23)=13.86, p<$ $.01]$, which, in turn, were more negative than COM exemplars $[6.4 \mu \mathrm{V} ; F(1,23)=16.45, p<.001]$. This is the same pattern as that observed in Experiment 1.

Late positive complex (500-900 $\mathrm{msec}$ ). The graded pattern of response to the three exemplar types continued beyond the N400 into the late part of the epoch. Mean amplitudes between 500 and $900 \mathrm{msec}$ over central and posterior sites were again analyzed using an ANOVA with 3 levels of exemplar type (COM, M-ONE, or M-TWO) and 15 levels of electrode site. There was a main effect of exemplar type $[F(2,46)=21.45, p<.0001, \varepsilon=1.00]$, with more negative responses to M-TWO than to M-ONE exemplars [3.6 vs. $5.0 \mu \mathrm{V} ; F(1,23)=15.22, p<.001]$ and more negative responses to M-ONE than to COM exemplars $[5.7 \mu \mathrm{V} ; F(1,23)=6.12, p<.05]$.

Summary. The featural similarity results fully replicated the patterns seen in Experiment 1. Between 150 and
$250 \mathrm{msec}$, a frontal component (likely the P2 and/or N2b) was more negative for the M-TWO exemplars than in the other two conditions. Following this, both the N400 (300$500 \mathrm{msec}$ ) and the LPC (500-900 msec) were graded by feature number, being most negative to the M-TWO exemplars and least negative to the COM exemplars.

Featural importance. Featural importance was examined by comparing exemplars missing a feature essential to object function (M-ESS) with exemplars missing a nonessential feature (M-NON). Statistical results confirm the visual impression (Figure 8) that M-ESS and M-NON exemplars do not differ in any time window (using the same analyses as above; all $F_{\mathrm{S}}<1.30$ ). ${ }^{3}$ Thus, in contrast to the pattern seen in Experiment 1, no effect of featural importance (measured in the difference between M-ESS and M-NON) was observed in the ERP data when the participants were making a parts detection rather than a category membership judgment. A 2 (experiment) $\times$ 2 (exemplar type: M-ESS or M-NON) $\times 26$ (electrode site) ANOVA confirmed an experiment $\times$ exemplar type interaction $[F(1,46)=4.19, p<.05]$ between 300 and $400 \mathrm{msec}$, with a larger difference between the exemplar types in Experiment 1 than in Experiment 2; this tendency was marginally significant between 400 and $500 \mathrm{msec}$ $[F(1,46)=2.63, p=.1]$ and was nonsignificant but in the same numerical direction thereafter.

\section{Discussion}

The effects of feature overlap that were observed in Experiment 1 were replicated in Experiment 2, in which the task was a parts detection judgment rather than a cat-
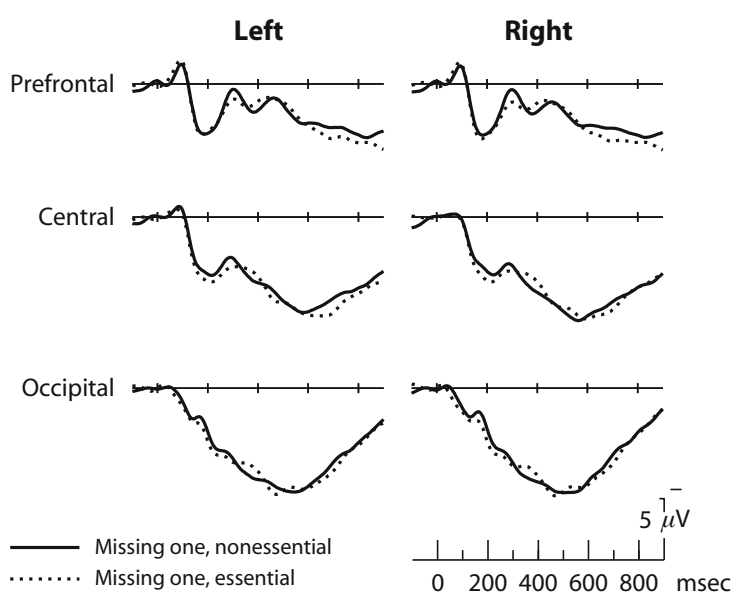

Figure 8. ERP effects of featural importance for Experiment 2. ERP responses to M-ESS and M-NON exemplars at six representative channels (filled-in shapes in Figure 3). Solid lines represent exemplars missing a feature that is nonessential to object function (M-NON), and dotted lines represent exemplars missing an essential feature (M-ESS). No condition-related differences were evident in the ERPs; in particular, the frontal positivity to M-ESS, as compared with M-NON, exemplars that was observed in Experiment 1 (cf. Figure 6) was not seen when the participants made a parts detection, rather than a category membership, judgment on the stimuli. 
egory membership judgment. Again, the presence of more novel featural information in the M-TWO condition was associated with an early perceptual/attentional effect that might reflect a reduced frontal P2 response and/or an enhanced N2b. Also as in Experiment 1, a negativity with a time course and distribution appropriate for an N400 effect was graded by number of features (most negative to M-TWO and most positive to COM exemplars). The fact that these effects were observed in a very similar form under two task conditions that made different demands on participants' attention, knowledge, and decision making is consistent with the view that assessments of featural similarity form an integral part of the representation and processing of object categories and that the N400 represents an automatic, task-invariant response to meaningful stimuli.

Replicating the findings of Lin and Murphy (1997), a clear influence of the functional significance of features was evident in the participants' behavioral responses. The participants were more accurate - and, in this case, also faster - in detecting the absence of a feature that was essential to object function than in detecting the absence of a nonessential feature, and this was true despite the fact that, across participants, identical exemplars occurred in both conditions and that knowledge about a feature's role was irrelevant to the task. However, different from Experiment 1, the ERP responses to these two types of exemplars (M-ESS and M-NON) were identical throughout the recording epoch. Thus, the frontal featural importance effect observed in Experiment 1 (which was tentatively linked to an N350 or an N2) seems to be task dependent and may arise only under conditions in which background knowledge is explicitly referenced (and, perhaps, leads to response conflict).

Because the ERPs did not reveal any differences between the M-ESS and M-NON conditions in Experiment 2, it is difficult to determine the roots of the behavioral knowledge-based effects that were observed. Although we cannot rule out the possibility of early knowledge-based effects that are not visible in scalprecorded ERPs, or of late effects that exceed the recording epoch, the data point to the possibility that during tasks such as parts detection, for which background knowledge is task irrelevant, the influence of functional significance may arise in processing stages beyond perceptual and semantic analyses - for example, through differential weightings of features in decision and/or response preparation stages. Given that average reaction times were over $1.5 \mathrm{sec}$, such effects may manifest in the ERP signal later than the 1-sec epoch used here and are likely to be temporally jittered with respect to stimulus onset (and thus difficult to pick up in the stimulus-locked ERP signal). ${ }^{4}$ However, whatever their source, it is noteworthy that the knowledge-based effects on behavior seen in the parts detection task do not seem to arise at the level of semantic processing (as indexed by the N400) and are not associated with the same electrophysiological signature as the effect seen when knowledge is explicitly called upon by the task, as in Experiment 1.

\section{GENERAL DISCUSSION}

The goal of this pair of experiments was to use ERPs to examine the functional roots of observed effects of background knowledge on categorization and object processing. In particular, we set out to test the hypothesis (e.g., Lin \& Murphy, 1997; Luhmann et al., 2006) that knowledge effects arise because the representation of objects and object categories in semantic memory includes rich structure, such as the functional significance of object features and causal relations between them. Such a view would predict that ERP measures of semantic access and processing, such as the N400, would be sensitive not only to factors highlighted by more traditional, similarity-based models, such as the number and diagnostic value of object features, but also to background knowledge.

To that end, we used stimuli (based on the set originally used by Lin and Murphy [1997]) in which the number of features an exemplar shared with its category was orthogonal to the importance of those features. The participants learned a set of novel object categories, each of which was associated with three emphasized features. They then evaluated test stimuli containing one, two, or three of those learned features; comparisons across these three item types allowed us to examine the attribute-matching processes that define traditional similarity-based categorization models. For those exemplars containing only two of the three learned features, the missing attribute was, for some participants, an essential feature whose absence rendered the object nonfunctional, but was, for other participants, a relatively superfluous feature that was not essential to the primary function of the object. In comparing responses to these stimuli (which, across participants, were perceptually identical), we could examine the role that knowledge-based information plays in the representation and online processing of objects under different task conditions.

\section{Electrophysiological Correlates of Similarity Evaluation}

Consistent with previous work, the pattern of overt responses revealed that the participants were highly sensitive to an exemplar's overall similarity to the trained exemplars: The likelihood of either endorsing an exemplar as a category member (Experiment 1) or considering it complete (Experiment 2) was affected by the number of learned features it contained. Correspondingly, in both experiments, analysis of the concurrently recorded ERPs revealed clear effects of feature overlap. In particular, a graded response to the number of expected features was seen on the N400, a well-studied component that has been linked to relatively implicit aspects of semantic access and integration (e.g., Kutas \& Federmeier, 2000; Olichney et al., 2000), and this pattern was comparable to similarity effects seen for well-learned semantic categories (e.g., Heinze et al., 1998). That the number of expected features produced task-invariant effects on this marker of semantic access suggests that similarity to learned exemplars (or to representations formed from learned exemplars) fa- 
cilitates the initial, automatic semantic evaluation of novel objects.

It is somewhat striking that newly learned object categories such as the ones in this set of experiments can elicit N400 effects at all. N400s have been previously observed to line drawings and pictures of familiar objects and are known to be affected by context, including that provided by a word cue (Ganis \& Kutas, 2003; Ganis, Kutas, \& Sereno, 1996; Sitnikova et al., 2003; West \& Holcomb, 2002). Thus, in principle, the type of stimuli used in the present experiment could be expected to elicit N400 modulations. However, the stimuli used in prior work involved objects and concepts that had been acquired over long spans of time and with which participants had had extensive experience. One study has also shown that during second-language learning, novel word forms can be relatively rapidly (after about $14 \mathrm{~h}$ of instruction) connected to concepts already stored in semantic memory (McLaughlin, Osterhout, \& Kim, 2004). To our knowledge, however, no prior study has reported N400 modulations to newly learned object representations in the context of newly learned labels for those objects. The appearance of N400 effects in our set of experimentsoccurring after only about 15 min of training - suggests that not only new words (McLaughlin et al., 2004) but also novel information about objects and fairly complex relations between their features can be rapidly integrated into semantic memory.

The graded effect of similarity observed on the N400 continued into later parts of the epoch (LPC), which in categorization work is associated with more explicit aspects of feature analysis and decision making (Azizian et al., 2006; Folstein \& Van Petten, 2004). This indicates that even in perceptually oriented tasks such as the parts identification task in Experiment 2, the analysis of individual features is not relegated to basic-level perceptual processing but consults semantic knowledge at several levels of analysis. An even earlier effect of attribute matching was evident in the $\mathrm{P} 2 / \mathrm{N} 2 \mathrm{~b}$ window $(150-250 \mathrm{msec})$, where stimuli missing two of the trained features (which had been replaced with altered features) were distinguished from other exemplar types. This effect suggests that a different distribution of attention and/or perceptual processing resources supports the apprehension of stimuli containing features that are incongruent with those associated with the object through training. That $\mathrm{P} 2 / \mathrm{N} 2 \mathrm{~b}$ responses distinguished between M-TWO and M-ONE, but not M-ONE and COMP, exemplars suggests that this effect may index novelty detection.

Thus, similarity - here operationalized as number of shared features - influenced processing at several levels, beginning with perceptual analysis and the allocation of visuospatial attention $(\mathrm{P} 2 / \mathrm{N} 2 \mathrm{~b})$, continuing through relatively early, implicit stages of access to meaning-based information (N400), and persisting into more explicit stages of processing (LPC), including behavioral responses. These influences of similarity were consistent across tasks that made different demands on the participants' attention, knowledge, and decision making. This relatively early and pervasive influence of similarity on processing is consistent with probabilistic models of categorization (e.g., Nosofsky, 1986; Rosch \& Mervis, 1975), which propose that natural categories are structured along the lines of feature overlap and that potential exemplars are evaluated by assessing the match between attributes of the exemplar and of stored exemplars and/or prototypes.

\section{Electrophysiological Correlates of Knowledge Effects}

Although attribute-matching processes have been well established, there is now substantial evidence that categorization judgments reflect more than a tallied checklist of independent features. In particular, background knowledge about an object's function and the role of its features with respect to that function has been shown to provide additional constraints on categorization decisions (for a review, see Murphy, 2002). We replicated behavioral results (Lin \& Murphy, 1997) showing that participants are less likely to consider objects missing a functionally significant feature as members of a learned category (Experiment 1) and are faster and more accurate to report that a feature is missing when that feature is essential to object functioning (Experiment 2). Thus, overt decisions about category membership and object parts are influenced by knowledge of each feature's significance, beyond its statistical relevance. Although background knowledge can affect even rapid decisions, the present set of experiments is the first to examine such processing with a functionally and temporally precise measure such as ERPs.

In Experiment 1, when the participants were likely to attend explicitly to knowledge-based information in the service of making judgments about category membership, an influence of the functional significance of features was observed in the ERP signal. Objects missing a nonessential feature elicited more negative potentials over the front of the head than did those missing an essential feature. This feature importance effect bears a resemblance to the N350, a component that has been linked to the ease of object identification/categorization (e.g., Schendan \& Kutas, 2002) and to the N2, a component that has been linked more generally to response conflict (Folstein \& Van Petten, 2008). The timing of the effect, beginning around $300 \mathrm{msec}$ after stimulus onset, shows that knowledgebased information can become available fairly early in the processing stream. However, the fact that this effect did not appear in Experiment 2, when participants determined whether or not an exemplar contained all of its learned features, shows that at least some types of knowledge effects are influenced by task demands.

Critically, however, in neither experiment did knowledge effects emerge on the N400. Thus, we found no evidence that knowledge effects arise because functionally significant features are differentially represented in and/ or accessed from long-term semantic memory. Of course, we cannot rule out semantic effects that might not manifest on the N400 (or on the later LPC, linked to more explicit aspects of semantic evaluation). However, it should be noted that the N400 has been shown to be sensitive to 
a wide range of low-level and high-level semantic factors (including repetition, frequency, association, similarity, typicality, and semantic context information in all modalities and ranging in complexity from single words through discourses and movies) on all meaningful stimulus types (including visual and auditory words, line drawings, pictures, gestures, faces, and environmental sounds; see Kutas, Federmeier, Staab, \& Kluender, 2007, for a review). Furthermore, in this set of experiments, the N400 was sensitive to the number of learned features. Thus, the results strongly suggest that background knowledge does not influence the access of information about objects from long-term semantic memory, at least for newly learned object categories, and that knowledge effects are dissociable from those related to the assessment of featural similarity, which does seem to affect semantic access.

It may be, instead, that knowledge-based information affects behavior by changing the salience of an object's parts and, thereby, how quickly a particular feature is processed and/or the amount of attention that is directed toward it. This view is consistent with work showing that categorization experience and expertise more generally can change the distinctiveness of object features (Goldstone, 1994; Goldstone et al., 2001) and affect how objects are processed perceptually (Johnson \& Mervis, 1997; Tanaka \& Curran, 2001). The (N350) effect observed in Experiment 1 might thus reflect such feature salience, as encoded in object templates (e.g., Schendan \& Kutas, 2007). Alternatively, or in addition, it may be that knowledge has its primary influence later in processing, during decision making. For example, one mechanism for knowledge effects considered by Lin and Murphy (1997) is that the functionally more important part is given greater weight in object-related decisions. Decisions based on that part are thus likely to be made more quickly and accurately. In some task conditions, such weighting could also create greater levels of response conflict, which might have manifested as increased N2 activity in Experiment 1. This view is consistent with the findings of the present pair of experiments, which suggest that all kinds of information about an object-including aspects that are more and less critical for object function - are encoded in semantic memory and accessed in qualitatively similar ways when that object is presented or referenced. The active use of this information to meet particular cognitive and behavioral goals, however, may then be critically shaped by what knowledge and experience suggest about its nature, salience, and task relevance.

\section{AUTHOR NOTE}

This research was supported by NIA Grant AG26308 to K.D.F. We thank the members of the Cognition and Brain Lab, especially the undergraduate assistants who helped with this project: Anastasia Kidd, Sarah Baisley, Jessica Atkins, and Michael Hawn. We thank Greg Murphy for sharing his stimuli, for commenting on an earlier draft of the manuscript, and for providing helpful advice throughout the project. Correspondence concerning this article should be addressed to K. D. Federmeier, Department of Psychology, University of Illinois, 603 E. Daniel St., Champaign, IL 61820 (e-mail: kfederme@illinois.edu).

\section{REFERENCES}

Azizian, A., Freitas, A. L., Parvaz, M. A., \& Squires, N. K. (2006). Beware misleading cues: Perceptual similarity modulates the N2/P3 complex. Psychophysiology, 43, 253-260.

Daffner, K. R., Mesulam, M. M., Scinto, L. F., Calvo, V., Faust, R., \& Hоцсомв, P. J. (2000). An electrophysiological index of stimulus unfamiliarity. Psychophysiology, 37, 737-747.

DALE, A. M. (1994). Source localization and spatial discriminate analysis of event-related potentials: Linear approaches. University of California, San Diego, Department of Cognitive Science.

Folstein, J. R., \& VAn Petten, C. (2004). Multidimensional rule, unidimensional rule, and similarity strategies in categorization: Eventrelated potential correlates. Journal of Experimental Psychology: Learning, Memory, \& Cognition, 30, 1026-1044.

Folstein, J. R., \& VAn Petten, C. (2008). Influence of cognitive control and mismatch on the N2 component of the ERP: A review. Psychophysiology, 45, 152-170.

Folstein, J. R., Van Petten, C., \& Rose, S. A. (2008). Novelty and conflict in the categorization of complex stimuli. Psychophysiology, 45, 467-479.

Ganis, G., \& Kutas, M. (2003). An electrophysiological study of scene effects on object identification. Cognitive Brain Research, 16, 123-144.

Ganis, G., Kutas, M., \& Sereno, M. I. (1996). The search for "common sense": An electrophysiological study of the comprehension of words and pictures in reading. Journal of Cognitive Neuroscience, 8, 89-106.

GoLDSTONE, R. L. (1994). Influences of categorization on perceptual discrimination. Journal of Experimental Psychology: General, 123, 178-200.

GoldStone, R. L., LiPPA, Y., \& SHIFFrin, R. M. (2001). Altering object representations through category learning. Cognition, 78, 27-43.

Heinze, H.-J., Münte, T.-F., \& Kutas, M. (1998). Context effects in a category verification task as assessed by event-related brain potential (ERP) measures. Biological Psychology, 47, 121-135.

Johnson, K. E., \& Mervis, C. B. (1997). Effects of varying levels of expertise on the basic level of categorization. Journal of Experimental Psychology: General, 126, 248-277.

Kutas, M., \& Federmeier, K. D. (2000). Electrophysiology reveals semantic memory use in language comprehension. Trends in Cognitive Sciences, 4, 463-470.

Kutas, M., Federmeier, K. D., Staab, J., \& Kluender, R. (2007). Language. In J. T. Cacioppo, L. Tassinary, \& G. Berntson (Eds.), Handbook of psychophysiology (3rd ed., pp. 555-580). Cambridge: Cambridge University Press.

Kutas, M., \& Hillyard, S. A. (1980a). Event-related brain potentials to semantically inappropriate and surprisingly large words. Biological Psychology, 11, 99-116.

Kutas, M., \& Hillyard, S. A. (1980b). Reading senseless sentences: Brain potentials reflect semantic incongruity. Science, 207, 203-205.

Kutas, M., \& Hillyard, S. A. (1983). Event-related brain potentials to grammatical errors and semantic anomalies. Memory \& Cognition, 11, 539-550.

Lin, E. L., \& MURPHY, G. L. (1997). Effects of background knowledge on object categorization and part detection. Journal of Experimental Psychology: Human Perception \& Performance, 23, 1153-1169.

LUCK, S. J., \& Hillyard, S. A. (1994). Electrophysiological correlates of feature analysis during visual search. Psychophysiology, 31, 291-308.

Luhmann, C. C., Ahn, W.-K., \& Palmeri, T. J. (2006). Theory-based categorization under speeded conditions. Memory \& Cognition, 34, 1102-1111.

McLaughlin, J., Osterhout, L., \& Kim, A. (2004). Neural correlates of second-language word learning: Minimal instruction produces rapid change. Nature Neuroscience, 7, 703-704.

Medin, D. L. (1989). Concepts and conceptual structure. American Psychologist, 44, 1469-1481.

MuRPHY, G. L. (2002). The big book of concepts. Cambridge, MA: MIT Press.

MurPhy, G. L., \& Medin, D. L. (1985). The roles of theories in conceptual coherence. Psychological Review, 92, 289-316. 
Nieuwenhuis, S., Yeung, N., van den Wildenberg, W., \& RidDERINKHOF, K. R. (2003). Electrophysiological correlates of anterior cingulate function in a go/no-go task: Effects of response conflict and trial type frequency. Cognitive, Affective, \& Behavioral Neuroscience, 3, 17-26.

Nobre, A. C., \& McCarthy, G. (1995). Language-related field potentials in the anterior medial temporal lobe: Modulation by word-type and semantic priming. Journal of Neuroscience, 15, 1090-1098.

NosofSKy, R. M. (1986). Attention, similarity, and the identificationcategorization relationship. Journal of Experimental Psychology: General, 115, 39-61.

Olichney, J. M., Van Petten, C., Paller, K. A., Salmon, D. P., Iragui, V. J., \& KutAs, M. (2000). Word repetition in amnesia: Electrophysiological measures of impaired and spared memory. Brain, 123, 1948-1963.

Palmeri, T. J., \& Blalock, C. (2000). The role of background knowledge in speeded perceptual categorization. Cognition, 77, B45-B57.

Pfefferbaum, A., Ford, J. M., Weller, B. J., \& Kopell, B. S. (1985). ERPs to response production and inhibition. Electroencephalography \& Clinical Neurophysiology, 60, 423-434.

Rosch, E., \& Mervis, C. B. (1975). Family resemblances: Studies in the internal structure of categories. Cognitive Psychology, 7, 573-605.

Schendan, H. E., \& Kutas, M. (2002). Neurophysiological evidence for two processing times for visual object identification. Neuropsychologia, 40, 931-945.

Schendan, H. E., \& Kutas, M. (2003). Time course of processes and representations supporting visual object identification and memory. Journal of Cognitive Neuroscience, 15, 111-135.

Schendan, H. E., \& Kutas, M. (2007). Neuropsychological evidence for the time course of activation of global shape, part, and local contour representations during visual object categorization and memory. Journal of Cognitive Neuroscience, 19, 734-749.

Sitnikova, T., Kuperberg, G., \& Holcomb, P. J. (2003). Semantic integration of videos in real-world events: An electrophysiological investigation. Psychophysiology, 40, 160-164.

SwaAb, T. Y., Brown, C., \& Hagoort, P. (1998). Understanding ambiguous words in sentence contexts: Electrophysiological evidence for delayed contextual selection in Broca's aphasia. Neuropsychologia, 36, 737-761.

TANAKa, J. W., \& Curran, T. (2001). A neural basis for expert object recognition. Psychological Science, 12, 43-47.

Tse, C. Y., Lee, C. L., Sullivan, J., Garnsey, S. M., Dell, G. S., FABIANI, M., \& GRATTON, G. (2007). Imaging cortical dynamics of language processing with the event-related optical signal. Proceedings of the National Academy of Sciences, 104, 17157-17162.

West, W. C., \& Holcomb, P. J. (2002). Event-related potentials during discourse-level semantic integration of complex pictures. Cognitive Brain Research, 13, 363-375.

WiSNIEWSKI, E. J. (1995). Prior knowledge and functionally relevant features in concept learning. Journal of Experimental Psychology: Learning, Memory, \& Cognition, 21, 449-468.

\section{NOTES}

1. To parallel the ERP analyses, response time analyses include both yes and no responses, but the pattern is unchanged if only the most frequent response type for each condition within each participant is used instead (yes to COM exemplars and no to M-ESS and M-TWO exemplars in all participants, and either yes $[n=7]$ or $n o[n=17]$ to M-NON exemplars).

2. The functional significance effect did not seem to be related to response choice, since the same pattern obtained for the participants who tended to endorse the M-NON items and those who tended to reject them.

3. There was also no difference between M-ESS and M-NON exemplars when all the trials (i.e., correct and incorrect) were included in the analysis, as in Experiment 1 (all $F_{\mathrm{s}}<1.67$ ).

4. Because the participants were allowed to blink and move their eyes after the first second of stimulus presentation, there are not enough artifact-free epochs to allow an examination of later effects in the ERP signal.

(Manuscript received July 4, 2008; revision accepted for publication December 6, 2008.) 